\title{
Beta delayed neutron measurements by means of Modular Total Absorption Spectrometer
}

\author{
Michat Stepaniuk ${ }^{1, *}$, Marek Karny ${ }^{1,2}$, Aleksandra Fijałkowska ${ }^{1,3}$, and Wojciech Bielewski ${ }^{1}$ \\ for MTAS Collaboration. \\ ${ }^{1}$ Faculty of Physics, University of Warsaw, PL-02-093 Warsaw, Poland \\ ${ }^{2}$ JINPA, Oak Ridge National Laboratory, Oak Ridge, Tennessee 37831, USA \\ ${ }^{3}$ Department of Physics and Astronomy, University of Tennessee, Knoxville, Tennessee 37966, USA
}

\begin{abstract}
Possibility to use Modular Total Absorption Spectrometer (MTAS) as a device to measure complete decay scheme, including $\beta$-n of neutron rich isotopes, has been investigated. Analysis of well known ${ }^{87} \mathrm{Br}$ with its $2.6 \%$ $\beta$-n branching ratio served as a test case. Preliminary results agree with the published data.
\end{abstract}

\section{Introduction}

Beta decay is one of the fundamental transformations of atomic nuclei. In most of the cases apart from ground state to ground state transformations, $\beta$ decay goes to the excited states in the daughter nuclei, followed by the $\gamma$-ray deexcitations. For nuclei with large enough Q-value $\beta$ decay to neutron unbound states becomes possible. In such cases neutron emission can be observed. Process is called $\beta$-delayed neutron emission $(\beta-n)$. Beta decay and in particular $\beta$-delayed neutron emission plays an important role in the astrophysical r-process $[1,2]$, and in the nuclear reactor physics. In the latter case not only criticality of the reactor depends on the $\beta$-n but also the cumulative fission yields which are used in many calculations, including decay heat calculations [3]. Therefore a good knowledge of decay schemes and $\beta$-n ratios are needed. Total absorption spectrometry is a technique that allows for a very efficient measurements of $\beta$-delayed $\gamma$-ray transitions [4-6].

In this contribution preliminary results of the analysis of the $\beta$ decay of ${ }^{87} \mathrm{Br}$ are presented. ${ }^{87} \mathrm{Br}$ is a well studied isotope with $\mathrm{T}_{1 / 2}=55.65(13) \mathrm{s}, \mathrm{Q}_{\beta}=6818(3) \mathrm{keV}$, and neutron emission probability $\mathrm{P}_{n}=2.60(4) \%$ [7]. Recent work of E.Valencia et al. [8] reports the total absorption studies of this isotope, with the striking conclusion that competition between $\beta$-delayed $\gamma$ rays and neutrons reaches levels $\approx 500 \mathrm{keV}$ above the neutron separation energy in ${ }^{87} \mathrm{Kr}\left(\mathrm{S}_{n}=5515.17(25) \mathrm{keV}[7]\right)$.

\section{Experimental setup}

The ${ }^{87} \mathrm{Br}$ was one of many uranium fission products, which decays have been measured in Holifield Radioactive Ion Beam Facility (HRIBF) at the Oak Ridge National Laboratory

\footnotetext{
*e-mail: michalstepaniuk@ student.uw.edu.pl
} 
(ORNL) [9] by means of Modular Total Absorption Spectrometer (MTAS) [6]. MTAS consists of $18 \mathrm{NaI}(\mathrm{Tl})$ hexagonal modules. Each of the 18 modules is 21 " long and 6.93" wide (side-to-side). There is also one central module of the same length and cross section, but with a 2.5 " hole drilled through. The crystals are arranged in a honeycomb like structure. Radioactive sample, to be measured, is placed between two $1 \mathrm{~mm}$ thick silicon detectors in the geometrical center of the detector. The total active $\mathrm{NaI}(\mathrm{Tl})$ mass is approximately one ton, making MTAS the largest and most efficient detector of this type currently in use. Due to its large volume $\beta$-n neutrons are efficiently slowed down and captured in the active volume of the detector, thus allowing for the estimation of the $\beta$-n component in the complete decay scheme [6].

Proton induced fission of ${ }^{238} \mathrm{UCx}$ target [10] was used to produce the nuclei of interest, which were further ionised in plasma ion-source and mass analysed by means of on-line mass separator. In case of A = 87 only bromine and krypton isotopes were present in the mass separated beam implanted on the mylar tape in front of the detector. After a pre-set collection time, radioactive samples were transported into the center of Modular Total Absorption Spectrometer and placed between two silicon detectors, serving as $\beta$ detectors. Signals from the detectors were processed using Pixie16 digital electronics modules [11]. Time gate for a single decay event was set to $500 \mathrm{~ns}$.

Experimental spectrum for mass $\mathrm{A}=87$ is presented in fig. 1 . It contains ${ }^{87} \mathrm{Br}$ with the admixture of ${ }^{87} \mathrm{Kr}$. ${ }^{87} \mathrm{Kr}$ was produced directly by the uranium fission and by the $\beta$ decay of ${ }^{87} \mathrm{Br} .{ }^{87} \mathrm{Kr}$ as a long lived isotope $\left(\mathrm{T}_{1 / 2}=76.3(5) \mathrm{min}\right)$ was independently measured with long irradiation (up to $30 \mathrm{~min}$ ) and long measurement $(115 \mathrm{~min}$ ) cycles, thus allowing to obtain the pure ${ }^{87} \mathrm{Kr}$ spectrum to be subtructed from the complex spectrum of fig. 1

Peak at $845 \mathrm{keV}$ was used to normalise the ${ }^{87} \mathrm{Kr}$ spectrum before the subtruction from ${ }^{87} \mathrm{Br}$ spectrum (fig. 1).

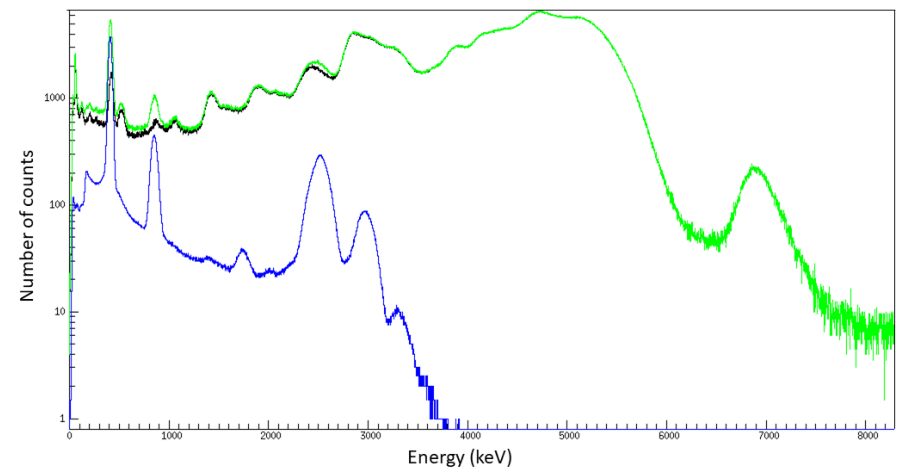

Figure 1. Experimental $\beta$ gated MTAS total energy deposit spectrum for mass $A=87$ (green), pure ${ }^{87} \mathrm{Kr}$ decay spectrum measured independently (blue), ${ }^{87} \mathrm{Br} \beta$ decay spectrum (black) obtained as a result of the spectra subtraction (green - blue). The ${ }^{87} \mathrm{Kr}$ spectrum was normalised on the $845 \mathrm{keV}$ peak.

\section{Simulations}

The code for MTAS response functions simulations was developed in the framework of Geant4 toolkit version 4.10.3.p01 [12]. The simulation code was previously used for numerous similiar analysis $[13,14]$ and is still under developement. 
Neutrons in MTAS are elasticly and non-elasticly scattered or captured (mostly in ${ }^{127} \mathrm{I}$ ). After neutron capture on ${ }^{127} \mathrm{I}, 6826.13 \mathrm{keV} \gamma$ rays are emmited [7], which are detected by the MTAS. Time needed by the neutrons to slow down to the energies which would allow its capture were investigated. Different initial neutron kinetic energies were investigated (200, $400,600,800 \mathrm{keV}$ and 1, 1.5, 2, 3, $4 \mathrm{MeV}$ ). Beginning of the time measurement was the the beginning of Geant 4 event and the end was the moment of the neutron capture. Ratios for neutrons captured in a given time (from zero to value of $t$ ) to total number of captured neutrons are presented in fig. 2. Percentages of neutron captures within the first $500 \mathrm{~ns}$ for all examined energies are above $90 \%$. This supports relevance of $500 \mathrm{~ns}$ time gate used in the data analysis.

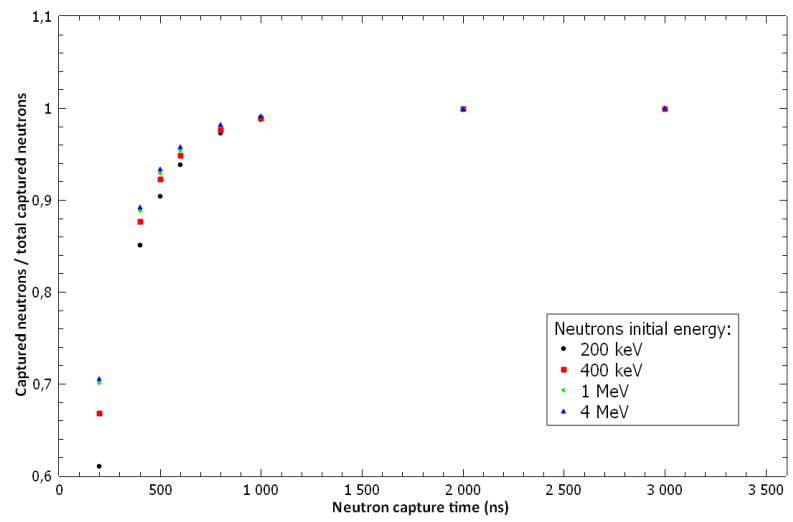

Figure 2. Ratios of neutrons captures in time (from zero to time $t$ ) to total number of neutrons captures. Simulated neutron initial kinetic energies: 200, 400, 1000, $4000 \mathrm{keV}$. Simulated MTAS efficiency for neutron capture varies from $42 \%$ at $0.2 \mathrm{MeV}$ to $17 \%$ at $4 \mathrm{MeV}$.

\section{Data analysis}

First step in the data analysis process was simulation of the expected MTAS total energy spectrum based on the data available in ENSDF database [7] (see blue curve in fig. 4). Then the existing $\beta$ feedings to all levels were changed to improve the agreement between the experimental and simulated spectrum. In many cases decay schemes derived from highresolution but low efficiency spectrometry are missing highly excited weakly fed levels. In order to account for this feeding, equally spaced pseudo-levels were added, which deexcitate by the $\gamma$ rays. In case of ${ }^{87} \mathrm{Br}$ the most important part of the data analysis was to include the neutron simulations in the combine evaluation process.

Simulated ${ }^{87} \mathrm{Br}$ neutrons spectrum is presented in fig. 3 together with total experimental spectrum. Peak at around $6.8 \mathrm{MeV}$ corresponds to events in which neutron was captured and all energies (both from slowing down and capture) was deposited in the detector. As can be seen in fig. 3 the FWHM of high-energy $\gamma$ rays are underestimated or high-energy neutrons are present in the decay of ${ }^{87} \mathrm{Br}$ which were not included in the ENSDF data file. The last possibility is unlikely as the $\mathrm{P}_{n}$ of ${ }^{87} \mathrm{Br}$ have been measured many times with various techniques and ${ }^{87} \mathrm{Br}$ is used as a calibration source for more exotic case studies.

In our preliminary data analysis the $\beta$-n branching ratio haven't been changed, although the width of the neutron peak have been adjusted. In addition pseudo-levels were added every $50 \mathrm{keV}$ between $5850-6300 \mathrm{keV}$ with deexcitation through single $\gamma$-ray to ground state as 


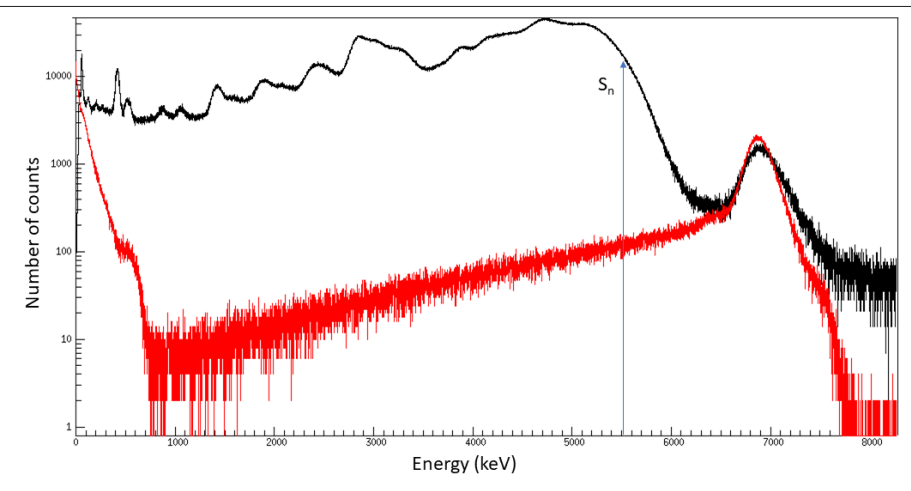

Figure 3. Simulation of the MTAS response function to the neutrons emitted after $\beta$ decay of ${ }^{87} \mathrm{Br}$ (red) in comparison with experimental MTAS spectrum of full ${ }^{87} \mathrm{Br} \beta$ decay (black). Spectra are normalized to the number of counts between 6500 and $7600 \mathrm{keV}$.

in existing decay scheme from $5473 \mathrm{keV}$ level upwards [7]. Results of the fit are shown in fig. 4.

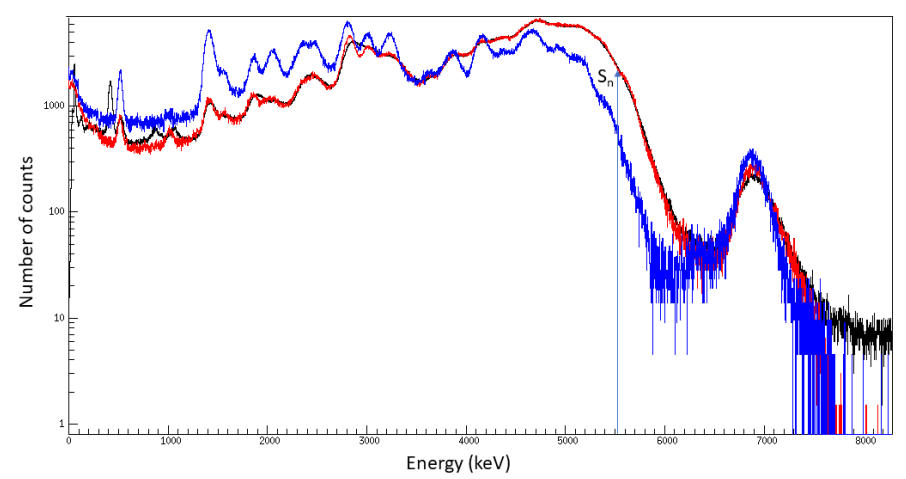

Figure 4. Experimental total energy deposited in MTAS detector emitted in the decay of ${ }^{87} \mathrm{Br}$ (black) compared with the simulated detector response based on ENSDF data (blue) and the simulated detector response based on the result of the analysis (red).

\section{Results discussion}

Current ENSDF data were used in ${ }^{87} \mathrm{Br}$ simulations and adjusted to fit experimental spectrum. Decay scheme based on high-resolution low-efficiency measurements overestimate lower energy states feeding and underestimate highly excited states transitions feeding. Levels above neutron separation energy $\left(S_{n}=5515.17(25) \mathrm{keV}\right)$ were added every $50 \mathrm{keV}$ up to $6300 \mathrm{keV}$. In accordance to current decay scheme, new levels deexcitate through single $\gamma$-ray to ground state. Final analysis will include different approach with other possible deexcitation patterns. The upper limit of $6300 \mathrm{keV}$ for added pseudo-levels is lower that the $6420 \mathrm{keV}$ used in recent work of E.Valencia et al. [8], but in presented analysis pseudo-levels up to $6.3 \mathrm{MeV}$ were sufficient to reconstruct the experimental spectrum. Featured preliminary result with 
$\beta-\gamma$ ratio above $S_{n}$ value of $4.75 \%$ is in good agreement with the value of $4.4 \%$ of Valencia et al. (calculated from the suplementary material).

In low energy part of the spectra, discrepancies between experimental and simulated spectra could be seen. Expected peaks from non-elastic neutrons scattering on ${ }^{127} \mathrm{I}$ are smeared in the simulated spectrum and neutrons response function at lowest energies is too intense when compared to experimental spectrum. These problems could be correlated with the way Geant4 is handling low energy neutrons and will be further investigated before final conclusion on the $\beta$ decay of ${ }^{87} \mathrm{Br}$ can be drawn.

\section{Acknowledgments}

We would like to thank the organizers of The XXII International Scientific Conference of Young Scientists and Specialists (AYSS-2018) for the oportunity to present this work and acknowledge the support of the Polish National Science Center under Contract No.2016/23/B/ST2/03559.

\section{References}

[1] J. Wu et al., Phys. Rev. Lett. 118, 072701 (2017)

[2] M. Madurga et al., Phys. Rev. Lett. 109, 112501 (2012)

[3] T. Yoshida and R. Nakasima, J. Nucl. Sci. Technol. 18, 393-407 (1981).

[4] L. Batist et al., Z. Phys. A351, 149 (1995)

[5] R. C. Greenwood et al., Nucl. Instrum. Methods Phys. Res. Sect. A 317, 175-184 (1992)

[6] M.Karny et al., Nucl. Instrum. Methods Phys. Res. Sect. A 836, 83-90 (2016)

[7] http://www.nndc.bnl.gov/ensdf/

[8] E. Valencia et al., Phys. Rev. C 95, 024320 (2017)

[9] J. R. Beene et al., J. Phys. G 38, 024002 (2011)

[10] D.W. Stracener et al., Nucl. Instrum. Methods Phys. Res. Sect. A 521, 126-135 (2004)

[11] XIA LLC, Hayward CA, (2009)

[12] J. Allison et al., IEEE Trans. Nucl. Sci. 53, 270-278 (2006)

[13] A. Fijałkowska, University of Warsaw PhD Thesis (2016)

[14] A. Fijałkowska et al., Phys. Rev. Lett. 119, 052503 (2017) 